Research Paper

\title{
NLS-RARa Inhibits the Effects of All-trans Retinoic Acid on NB4 Cells by Interacting with P38a MAPK
}

\author{
Chunlan Xiao', Liang Zhong ${ }^{2}$, Zhiling Shan ${ }^{2}$, Ting $\mathrm{Xu}^{2}$, Liugen Gan², Hao Song ${ }^{2}$, Rong Yang ${ }^{2}$, Liu Li ${ }^{2}$ and \\ Beizhong Liu ${ }^{1,2}$ \\ 1. Central Laboratory of Yong-chuan Hospital, Chongqing Medical University, Chongqing 402160, China; \\ 2. Key Laboratory of Laboratory Medical Diagnostics, Ministry of Education, Department of Laboratory Medicine, Chongqing Medical University, \\ Chongqing, 400016, China. \\ $\square$ Corresponding author: Bei-Zhong Liu, Department of Laboratory Medicine, Chongqing Medical University, 1\#, Yixueyuan Road, Chongqing, 400016, China. \\ Tel: +86 18716474304, Fax: +86 023-68485006; E-mail: liubeizhong@cqmu.edu.cn.
}

(1) Ivyspring International Publisher. Reproduction is permitted for personal, noncommercial use, provided that the article is in whole, unmodified, and properly cited. See http://ivyspring.com/terms for terms and conditions.

Received: 2016.02.25; Accepted: 2016.07.07; Published: 2016.07.18

\begin{abstract}
Nuclear localization signal retinoic acid receptor alpha(NLS-RARa), which forms from the cleavage of promyelocytic leukemia-retinoic acid receptor alpha(PML-RAR $\alpha$ ) protein by neutrophil elastase(NE), possesses an important role in the occurrence and development of acute promyelocytic leukemia(APL). However, the potential mechanism underlying the effects of NLS-RARa on APL is still not entirely clear. Here, we investigated the effects of NLS-RARa on APL NB4 cells and its mechanism. We found that all-trans retinoic acid(ATRA) could promote differentiation while inhibit proliferation of APL NB4 cells via upregulating the expression of phosphorylated $p 38 \alpha$ mitogen-activated protein kinase(p-p38 $\alpha$ MAPK). We also found that NLS-RARa could inhibit differentiation while accelerate proliferation of NB4 cells via downregulating the expression of $\mathrm{p}-\mathrm{p} 38 \mathrm{\alpha}$ protein in the presence of ATRA. Furthermore, immunofluorescence and co-immunoprecipitation assays confirmed NLS-RAR $\alpha$ interacted with p38a protein directly. Finally, application of PD 169316, an inhibitor of $\mathrm{p} 38 \alpha$ protein, suggested that recruitment $p 38 \alpha-c o m b i n d e d ~ N L S-R A R \alpha$ by ATRA eventually caused activation of $p 38 \alpha$ protein. In summary, our study demonstrated that ATRA cound promote differentiation while inhibit proliferation of APL NB4 cells via activating $p 38 \alpha$ protein after recruiting $p 38 \alpha-c o m b i n d e d$ NLS-RAR $\alpha$, while NLS-RAR $\alpha$ could inhibit the effects of ATRA in the process.
\end{abstract}

Key words: acute promyelocytic leukemia, NB4 cells, all-trans retinoic acid, nuclear localization signal retinoic acid receptor alpha, p38a MAPK.

\section{Introduction}

Acute promyelocytic leukemia (APL) is a normal type of acute myeloid leukemia (AML), in which leukemia cells possess the ability to infinitely proliferate. Moreover, cell differentiation in APL is suppressed at immature stages due to the fusion protein, PML-retinoic acid receptor alpha(PML-RARa)[1, 2], a strong transcriptional repressor for genes involved in granulocyte differentiation[3]. PML-RARa is formed by the chromosomal translocation of the RARa gene on chromosome 17 to the PML gene on chromosome 15[4]. It has been found that neutrophil elastase (NE) in early APL cells can cleave PML-RARa into two mutational proteins, PML (NLS(-)) and nuclear localization signal NLS-RARa (Figure 1), which was significant for the development of APL $[5,6]$. Previous researchers have studied the function of the mutational protein, NLS-RARa, and have verified that it can accelerate the proliferation of NB4 cells while inhibit differentiation of HL60 cells [7, 8]. Moreover, the function of NLS-RARa proved to be linked with Akt signaling pathway [8].

MAPK family members and Akt signaling pathway played crucial roles in the clonal formation 
of KG1a cells, which mimic a CD34+ cell model [9]. In addition to hematological tumors, researchers have found that p38 MAPK and Akt pathways played significant roles in myogenesis and muscle differentiation [10-12]. Furthermore, it has been demonstrated that transcription activity of RARa on target genes decreased when it directly interacted with p38a MAPK in the presence of ATRA [13], which is a drug used to treat APL [14].

Thus, we speculated that the activity of p38a MAPK may influence the proliferation and differentiation of APL NB4 cells, and the effects of NLS-RARa on differentiation and proliferation of NB4 cells may be related to the activity of p38a MAPK. Then we explored the potential mechanism underlying the effects of NLS-RARa on NB4 cells.

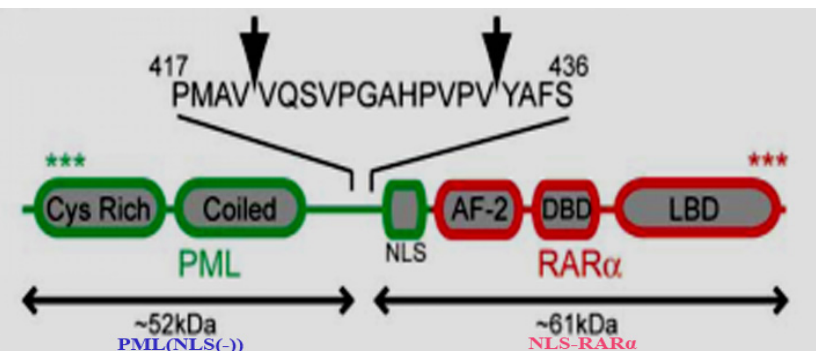

Figure 1. Identification of NE cleavage sites in PML-RARa[5].Arrows indicate the position of NE cleavage within the PML portion of PML-RARa, after V420 and V432. The approximate expected sizes of the peptide fragments generated by these cleavage events are shown. Several known domains in PML-RAR $\alpha$ are labeled: cystine-rich RING/B Box domain(Cys Rich), helical coiled-coil domain(Coiled), nuclear localization signal(NLS), transcriptional activation domain(AF-2),DNA binding domain(DBD), and the ligand binding domain(LBD).

\section{Materials and Methods}

\section{Cell lines}

APL cell line NB4 cells, NB4 cells infected with lentivirus only(LV-NC-NB4) and NB4 cells infected with NLS-RARa-lentivirus(LV-NLS-RARa-NB4) cells were saved by our own laboratory, and cultured in RPMI-1640 medium supplemented with $10 \%$ fetal bovine serum(FBS; Gibco, Australia) in an environment with $5 \% \mathrm{CO} 2$ at $37^{\circ} \mathrm{C}$.

293T cells were saved by our own laboratory and cultured in DMEM medium supplemented with $10 \%$ fetal bovine serum (FBS; Gibco, USA) in an environment with $5 \% \mathrm{CO} 2$ at $37^{\circ} \mathrm{C}$.

\section{CCK-8 assay}

Cell proliferation was quantified by CCK-8 kit (7Sea Cell Counting Kit; Sevenseas Futai Biotechnology Co., Ltd.,Shanghai, China). Cells in each group were seeded in 96-well plates at a density of 5000 cells/well. Then cells were incubated with various of treatments for 3 days. In brief, $10 \mu \mathrm{l}$ of CCK-8 assay was added to each well followed by incubation for $1 \mathrm{~h}$ at $37^{\circ} \mathrm{C}$. The cell proliferation was assessed by detection of absorbance at $450 \mathrm{~nm}$ using a spectrophotometer. The optical density value is positive correction with cell proliferation.

\section{Western blot}

Cells in each group were washed with ice-cold phosphate-buffered saline (PBS) three times and lysed in RIPA solution containing protease inhibitor phenylmethanesulfonyl fluoride (PMSF), phosphatase inhibitor $\mathrm{NaF}$ and $\mathrm{Na} 3 \mathrm{VO} 3$. Protein concentration was measured by BCA method. $50 \mu \mathrm{g}$ total protein was added in $10 \%$ sodium dodecyl sulfate-polyacrylamide gel and then transfered to nitrocellulose membranes. The membranes were blocked with 5\% non-fat milk for 1 hour and incubated with specific antibodies(polyclonal antibody against p-p38a MAPK; 1:1000; Millipore, USA; polyclonal antidoby against p38a MAPK, HA-Tag; 1:1000; CST, USA; monoclonal antibody against Myc-Tag; 1:1000; CST, USA; polyclonal antibody against RARa; 1:1000; Santa Cruz, USA; polyclonal antibody against C/EBP $\beta, C D 11 b ; 1: 500$; Wanleibio; China) overnight at $4{ }^{\circ} \mathrm{C}$ and then with secondary antibody(goat anti-rabbit antibody, 1:5000 and goat anti-mouse antibody, 1:2000; Zhongshan Goldenbridge Biotechnology Co. Ltd., Beijing, China) for $1 \mathrm{~h}$ at $37^{\circ} \mathrm{C}$. After washing with Tris-Buffered Saline Tween-20 and Tris-Buffered Saline (TBST and TBS), the autoradiograms were scanned and subjected to densitometry. $\beta$-actin (monoclonal antibody against $\beta$-actin, 1:1000; Zhongshan Goldenbridge Biotechnology Co. Ltd.,Beijing, China) was used as an internal control.

\section{Construction of eukaryotic expression vectors of pCMV-Myc-p38 $\alpha$ and LV-NLS-RAR $\alpha$}

Primer sequences of p38a(forward: TAA CTCGAG TAA TGT CTC AG G AGA GGC CCA CGT; reverse: TAT TAA GCGGCCGC TCA GGA CTC CAT CTC TTC TTGG) were designed by Primer-Premier 5.0 and synthesized by Sangon Biotech company. Underlined sequences are Restriction Enzyme cutting sequences (Xho1; Not1). cDNAs synthesized from RNA which was extracted from APL NB4 cells were used as PCR(Polymerase Chain Reaction) templates. Reaction system components: PrimeSTARTMHS(Premix)(TaKaRa, Japan), cDNAs, primers of p38a and $\mathrm{ddH}_{2} \mathrm{O}$. The PCR conditions were: pre-denaturation at $95^{\circ} \mathrm{C}$ for $5 \mathrm{~min}$, 29 cycles of denaturation at $98^{\circ} \mathrm{C}$ for $10 \mathrm{~s}$, annealing at $68.8^{\circ} \mathrm{C}$ for $30 \mathrm{~s}$, and extension at $72{ }^{\circ} \mathrm{C}$ for $80 \mathrm{~s}$, and a final extension at $72{ }^{\circ} \mathrm{C}$ for $5 \mathrm{~min}$. PCR product was CDS of p38a with 1083 bp. Purified products with E.Z.N.A Gel Extraction kit(OMEGA, USA), digested 
products and pCMV-Myc vector with Restriction Enzyme Xho1 and Not1(Xho1, Xho1; NEB, England), connected products of p38a to vector with T4 DNA ligase (TaKaRa, Japan). Then transformated connected products into competence DH5a and amplified by bacteria culture. After sequences were proved accurate, Q-PCR (quantitative polymerase chain reaction) and western blot verified pCMV-Myc-p38a MAPK expression plasmid.

Construction of LV-NLS-RARa was as described [8].

\section{Co-immunoprecipitation assay}

The binding activity of proteins was determined by co-immunoprecipitation (Co-IP) assay. For this study, total cell lysates were incubated with the desired antibodies for $16 \mathrm{~h}$ at $4{ }^{\circ} \mathrm{C}$ and the immuno-complex was collected on Protein A/G PLUS-Agarose(Santa Cruz, USA) for $5 \mathrm{~h}$ and washed 3 times with lysis buffer prior to boiling in SDS sample buffer. Immunoprecipitated proteins were separated on SDS-polyacrylamide gels and transferred to nitrocellulose membranes for Western blot analysis.

\section{Indirect immunofluorescence assay}

The localization between p38a protein and NLS-RARa was confirmed by indirect immunofluorescence assay. LV-NLS-RARa-NB4 cells suspension were collected, centrifuged and washed by PBS for three times. Cells on glass coverslips were fixed with $4 \%$ paraformaldehyde for 20 minutes. Subsequently, cells were permeabilized and blocked respectively with $0.1 \%$ Triton X-100(in PBS) and 10\% goat serum (in PBS) for 30 minutes at room temperature. For immunofluorescence staining, the rabbit polyclonal antibody against $\mathrm{p} 38 \mathrm{a}$ protein, mouse monoclonal antibody against HA-tag(p38a, HA-tag; 1:100; CST, USA) were used to probe p38a protein and NLS-RARa(as NLS-RARa was inserted to eukaryotic expression vector of pCMV-HA) overnight at $4{ }^{\circ} \mathrm{C}$ and goat against rabbit-IgG-TRITC, goat against mouse-IgG-FITC(rabbit-IgG-TRITC, mouse-IgG-FITC; 1:200; Zhongshan Goldenbridge Biotechnology Co. Ltd., Beijing, China) was used to detect rabbit and mouse IgG for $1 \mathrm{~h}$ at room temperature. Finally, nuclei were stained by DAPI (Beyotime; 1:10) at room temperature for $5 \mathrm{~min}$. All the coverslips were washed with PBS for three times. The coverslips were immobilized on the glass slides by $70 \%$ glycerol in PBS and viewed under a fluorescence microscope (Nikon, Tokyo, Japan).

\section{Dual luciferase reporter assay}

$293 \mathrm{~T}$ cells were transiently transfected with $0.4 \mu \mathrm{g}$ pTK-Luc reporter plasmids, $0.01 \mu \mathrm{g}$
pRLtk(Promega), and 0.3 $\mathrm{g}$ expression vectors per well of 24 wells plate when cell confluence was about $70 \%$. Cells were cultured in medium containing $10 \%$ FBS for 6 hours and then the medium was replaced. After $24 \mathrm{~h}$ of transfection (LipoFiterTM Liposomal Transfection Reagent; HANBIO, China), cells were treated for a further $24 \mathrm{~h}$ with $10 \mathrm{nM}$ ATRA, then cells were lysed and normalized luciferase activities were determined.

\section{Statistical analysis}

All the data were presented as the mean \pm SD. Student's t-test was applied for the statistical analysis of three independent groups by GraphPad Prism 5 software. For all tests, ${ }^{*} p<0.05$ or ${ }^{* *} p<0.01$ was considered statistically significant. NS was considered no statistically significant.

\section{Results}

\section{Promotion of differentiation while suppression of proliferation of NB4 cells correlated with the activation of $\mathrm{p} 38 \alpha$ protein by ATRA}

It has been shown that ATRA inhibits differentiation of APL cells while accelerate their proliferation. Moreover, the effects of ATRA have been correlated with activation of p38a MAPK [13, 15, 16]. To verify this, and to discover an ATRA concentration that is related to both the biological function(differentiation and proliferation) and the activity of p38a protein in NB4 cells, we first examined the expressions of p-p38a and p38a proteins after treating NB4 cells with ATRA (physiological concentration: $10 \mathrm{nM}$ and pharmacological concentration: $1 \mu \mathrm{M}$ ) for 3 days. We found that the expression of p38a protein was maintained, regardless of ATRA treatment. However, the expression of $\mathrm{p}-\mathrm{p} 38 \mathrm{a}$ protein increased obviously in the experimental group compared to the negative control (dimethylsulfoxide(DMSO)-treated) group, especially when cells were treated with $10 \mathrm{nM}$ ATRA (Figure 2A and 2B). We further determined the expressions of $\mathrm{C} / \mathrm{EBP} \beta$, a myeloid differentiation marker protein $[17,18]$, and CD11b, a surface myeloid differentiation marker protein $[13,19,20]$. We found that the expressions of $\mathrm{C} / \mathrm{EBP} \beta$ and $\mathrm{CD} 11 \mathrm{~b}$ proteins were significantly increased after treating NB4 cells with ATRA (Figure 2A, 2C, and 2D). Moreover, changing trend of $\mathrm{C} / \mathrm{EBP} \beta$ protein paralleled that of p-p38a protein. Based on the above results, we surmised that the differentiation of NB4 cells related to the activity of p38a protein. Next, we determined NB4 cell proliferation with cell counting kit and observed morphological characteristics with an inverted microscope (Figure 2E and 2F). As expected, 
both concentrations of ATRA inhibited NB4 cell proliferation. Taken together, the above results suggested that not only the differentiation but also the proliferation of NB4 cells correlated with the activation of p38a protein by ATRA.

\section{Promotion of cell differentiation while suppression of cell proliferation resulted from activation of $\mathrm{p} 38 \alpha$ protein by ATRA}

We have known that RARa could regulate the expressions of genes involved in cell differentiation and proliferation after binding to retinoid-responsive elements (RARE) in genes [21, 22, 24-26]. However, p38a protein can interact with RARa directly to inhibit the transcriptional activity of RARa in the presence of ATRA [13]. Thus, we speculated that p38a protein could regulate the expressions of genes involved in cell differentiation and proliferation similar to RARa. We first constructed a eukaryotic expression plasmid of pCMV-Myc-p38a and tested the availability of the retinoid-responsive reporter plasmid, pTK-Luc, offered by Professor Dmitrii Kamashev and his partners in France [23]. The eukaryotic expression plasmid of pCMV-Myc-p38a was successfully constructed (Figure $3 \mathrm{~A}-3 \mathrm{~B}$ ) and pTK-Luc plasmid was proved to be available (Figure $3 C)$.

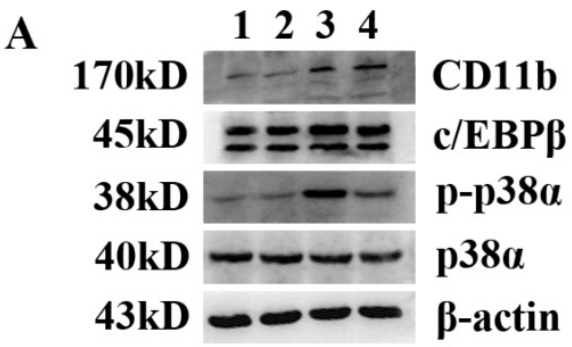

$\mathbf{B}$

C

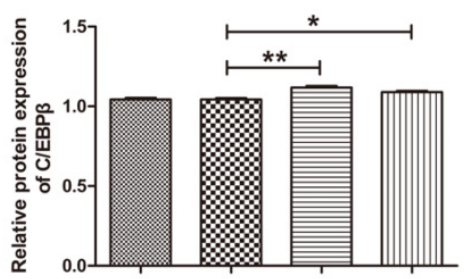

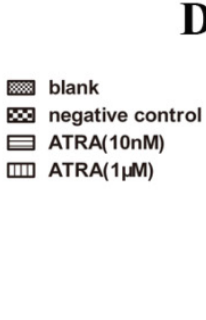

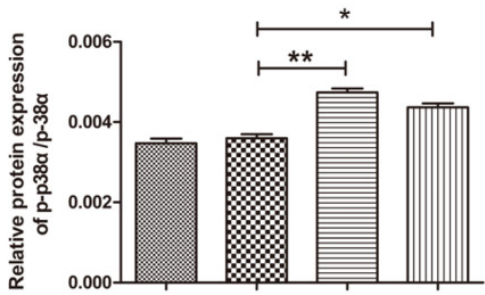

blank

negative contro

寻RA(10nM)

四 ATRA $(1 \mu M)$

D

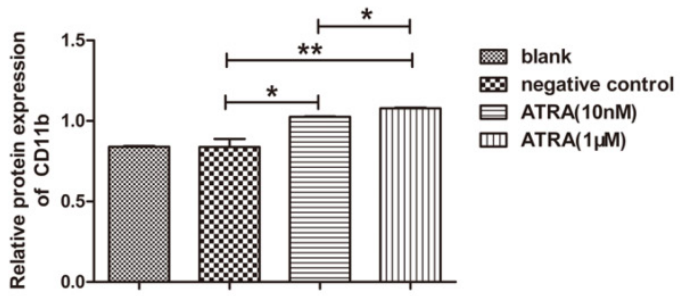

$\mathbf{E}$

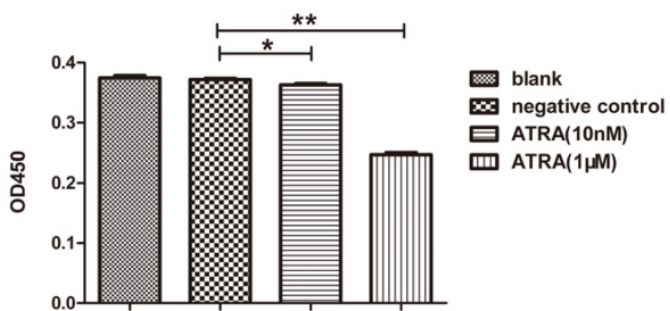

$\mathbf{F}$
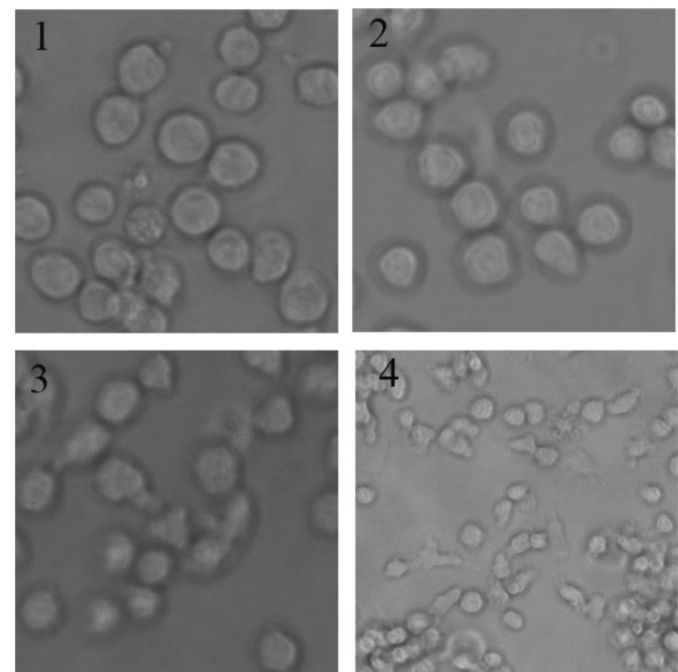

Figure 2. Promotion of differentiation while suppression of proliferation of NB4 cells correlated with the activation of p38a protein by ATRA. (A) Western blot analysis of the expressions of $\mathrm{p} 38 \alpha$, p-p38a, differentiation makers, C/EBP 3 and CD1 lb, in NB4 cells cultured with ATRA for 3 days; (B-D) Quantitative analysis of the expression levels of $\mathrm{P}-\mathrm{p} 38 \mathrm{a} / \mathrm{p} 38 \mathrm{a}, \mathrm{C} / \mathrm{EBP} \beta, \mathrm{CD} 1 \mathrm{lb}$ after normalization with $\beta$-actin; (E) NB4 cells were treated with ATRA for 3 days. Then the optical density value at 450nm(OD450) was measured by CCK-8 assay; (F) NB4 cells were treated with ATRA for 3 days. Then Observed morphological characteristics using an inverted microscope. All data are presented as mean \pm SD. $* \mathrm{p}<0.05, * * \mathrm{p}<0.01$. (1: blank group(non-manipulated); 2: negative group(dimethylsulfoxide(DMSO)-treated); 3: experimental group treated with 10 nM ATRA; 4: experimental group treated with $1 \mu$ M ATRA) 
A

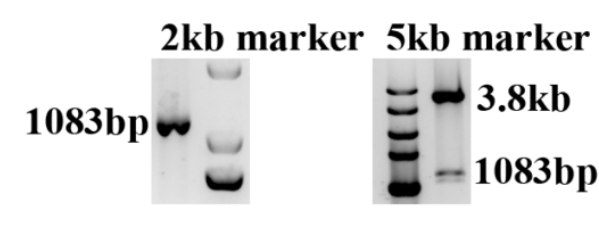

C

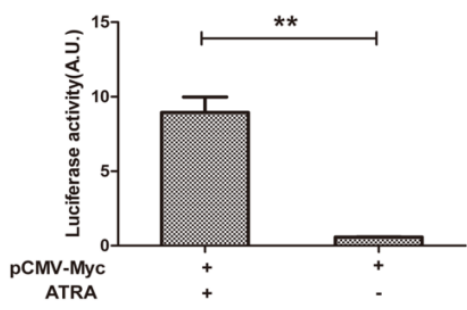

$\mathbf{E}$

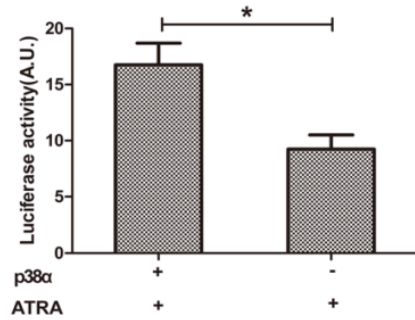

B

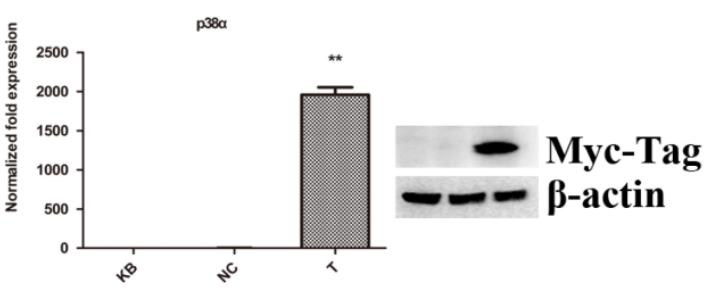

D

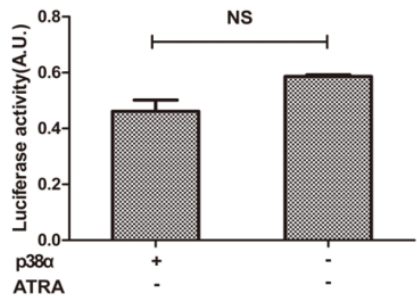

p38a +-

ATRA + +

Figure 3. Promotion of cell differentiation while suppression of cell proliferation resulted from activation of p38a protein by ATRA. (A) PCR combined with restriction enzyme digestion analysis of p38a gene; (B) RT-qPCR and Western blot analysis of the expression of Myc-tagged p38a gene; (C, D, E) 293T cells were transfected with pCMV-Myc-p38a or pCMV-Myc plasmids $(0.3 \mu \mathrm{g})$, retinoid-responsive reporter plasmid, pTK-Luc $(0.4 \mu \mathrm{g})$, and pRLtk plasmids $(0.01 \mu \mathrm{g})$. $24 \mathrm{hours}$ later, cells were treated with $10 \mathrm{nM}$ ATRA or vehicle for another 24 hours. Then cells were lysed and normalized luciferase activities were determined. All data were presented as mean $\pm S D$. $* p<0.05$, $* * p<0.01, N S:$ not statistical significant. (KB group: 293T cells were non-manipulated, NC group: 293T cells were transfected with pCMV-Myc plasmids, T group: 293T cells were transfected with pCMV-Myc-p38a plasmid).

Next, We co-transfected 293T cells with plasmids and then treated cells with ATRA or vehicle. As expected, changes of luciferase activity in two groups had no statistical significance when cells treated with vehicle (Figure 3D). However, when cells treated with ATRA, luciferase activity and the expression of p-p38a protein remarkably increased compared to the negative control group (Figure 3E). It suggested that promotion of cell differentiation while suppression of cell proliferation resulted from activation of p38a protein rather than resulted in activation of $p 38 a$ protein.

\section{ATRA application led to NLS-RAR $\alpha$-induced differential inhibition while accelerated proliferation of NB4 cells via down regulating p-p38 $\alpha$ protein level}

We next explored the effects of NLS-RARa on NB4 cells and its underlying mechanism. First, we established an early APL cell model through lentivirus-mediated overexpression of NLS-RARa in NB4 cells (Figure 4A). Then, Western blot detected the expressions of various proteins. Compared to the negative control group, the expressions of p38a and p-p38a proteins maintained (Figure 4B, 4D), while the expressions of differentiation markers, $\mathrm{C} / \mathrm{EBP} \beta$ and $\mathrm{CD} 11 \mathrm{~b}$ decreased (Figure 4C, 4E-4F). We have observed that the effects of p-p38a protein on differentiation and proliferation of NB4 cells were related to ATRA. Thus, we next detected the expressions of various proteins and cell proliferation after treating cells with $10 \mathrm{nM}$ ATRA for 3 days. Compared to the negative control group, the expressions of $\mathrm{p}-\mathrm{p} 38 \mathrm{a}, \mathrm{C} / \mathrm{EBP} \beta$, and $\mathrm{CD} 11 \mathrm{~b}$ proteins decreased while the expression of p38a protein maintained (Figure 4B). Cell proliferation accelerated when compared to the negative control group (Figure $4 \mathrm{G})$. These data suggested that NLS-RARa could inhibit differentiation while accelerate proliferation of NB4 cells via down regulating p-p38a protein level in the presence of ATRA. These data also implied that NLS-RARa could inhibit differentiation of NB4 cells via an alternative pathway in the absence of ATRA. 


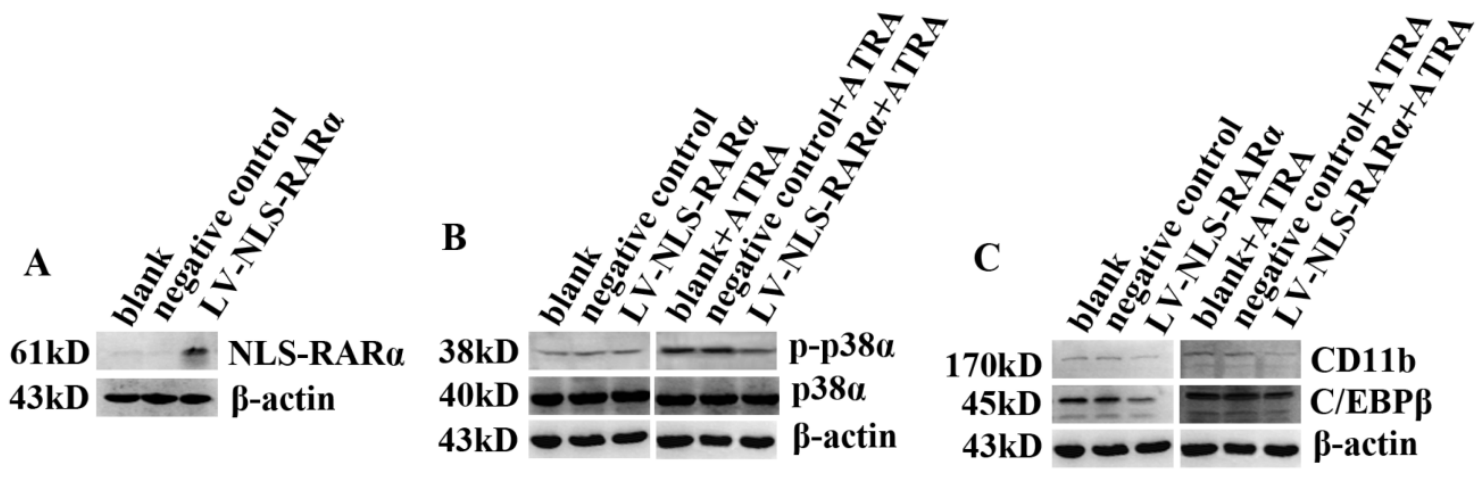

D

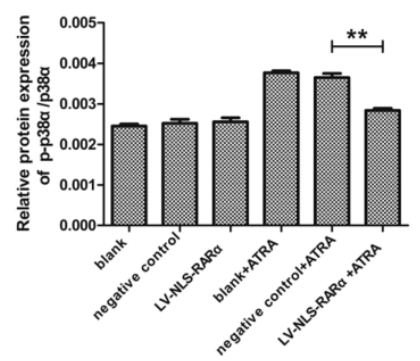

E

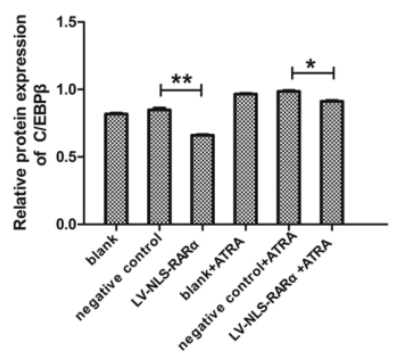

F

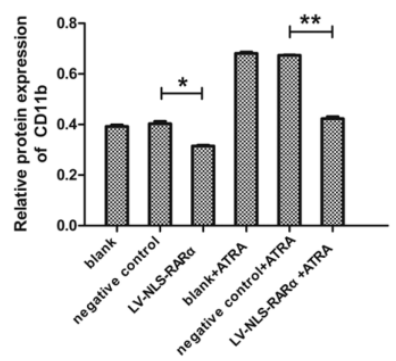

G

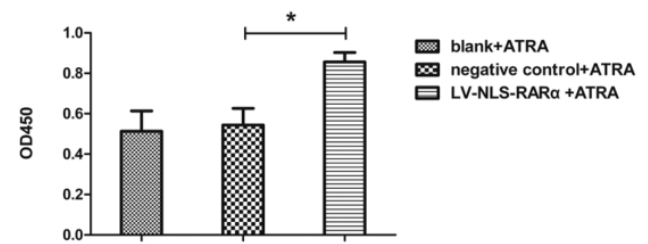

Figure 4. ATRA application led to NLS-RARa-induced differential inhibition while accelerated proliferation of NB4 cells via down regulating P-p38a protein level. (A)Western blot analysis of the overexpression of NLS-RAR $\alpha$ in NB4 cells; $(\mathbf{B}, \mathbf{C})$ Western blot analysis the expressions of p38 $\alpha, \mathrm{p}-\mathrm{p} 38 \alpha, \mathrm{C} / \mathrm{EBP} \beta$ and CD1 lb proteins in NB4 cells after treating cells with/without $10 \mathrm{nM}$ ATRA for 3 days; (D-F) Quantitative analysis of the expression levels of $p$-p38 $\alpha / p 38 \alpha, C / E B P \beta, C D 11 \mathrm{~b} p$ roteins after normalization with $\beta$-actin; (G) NB4 cells were treated with $10 \mathrm{nM}$ ATRA for 3 days. Then the optical density value at $450 \mathrm{~nm}(\mathrm{OD} 450)$ was measured by CCK-8 assay. All data are presented as mean \pm SD. $*_{p}<0.05, p<0.01$.(blank group: non-manipulated, negative control group: lentivirus only).

\section{Downregulation of p-p38a protein level may be induced by a direct interaction between NLS-RAR $\alpha$ and $p 38 \alpha$ protein}

To investigate the underlying mechanism by which NLS-RARa induced downregulation of p-p38 $\alpha$ protein level, we carried out an indirect immunofluorescence assay. As shown in Figure 5A, cells appeared yellow, indicating NLS-RARa (green) merged with p38a protein (red). These findings suggested close localization of NLS-RARa and p38a protein in three-dimensional space. However, this assay couldn't distinguish whether it's indirect or direct interaction between them. Therefore, we performed a co-immunoprecipitation experiment in 293T cells. Immunoprecipitation with an anti-RARa antibody resulted in co-precipitation of p38a protein (Myc-tagged) only in cells that overexpressed both proteins (Figure 5C). This interaction was confirmed when NLS-RARa (HA-tagged) instead of p38a protein, was co-immunoprecipitated in complementary experiments (Figure 5D).

\section{Recruitment p38 $\alpha$-combinded NLS-RAR $\alpha$ by ATRA eventually caused activation of $p 38 \alpha$ protein}

We have demonstrated that ATRA could promote differentiation while inhibit proliferation of NB4 cells via activating p38a protein. We also found NLS-RARa could inhibit those effects of ATRA by interacting with $\mathrm{p} 38 \mathrm{a}$ protein directly. However, it is faint ATRA directly activates p38a protein and then recruits NLS-RARa or recruitment p38a-combinded NLS-RARa by ATRA eventually causes activation of p38a protein. In order to clarify this question, we investigated the effects of an inhibitor of p38 protein, PD169316 (MCE, USA). We found that PD169316 (10 $\mu \mathrm{M})$ inhibited the effects of ATRA on 
NLS-RARa-overexpressed NB4 cells incluing an increase of the expressions of $p$-p38a, $C / E B P \beta$ and CD11b(Figure 6A). It also inhibited the effect of ATRA on NLS-RARa-overexpressed NB4 cell proliferation (Figure 6B). However, it didn't change the effect of ATAR on the expression of NLS-RARa in NLS-RARa-overexpressed NB4 cells (Figure 6A). Moreover, PD169316 (10 $\mu \mathrm{M})$ couldn't block the direct interaction between NLS-RARa and p38a protein (Figure 6C). Taken together, it suggested that direct interaction between NLS-RARa and p38a protein was naturally existed in cells no matter whether p38a protein was activated or not. Taking this one step further, ATRA recruited p38a-combinded NLS-RARa then caused activation of $\mathrm{p} 38 \mathrm{a}$ protein.

A

anti-HA-Tag/FITC

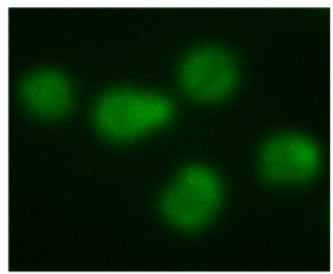

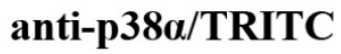

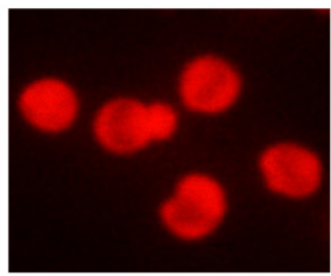

merged

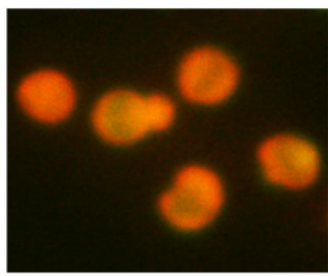

B

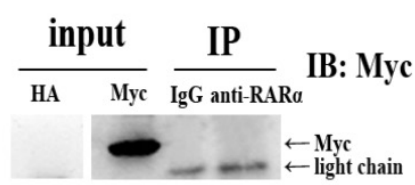

C

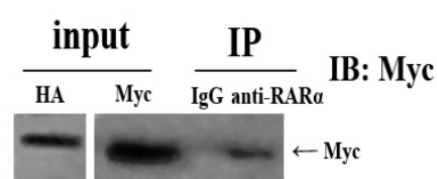

D

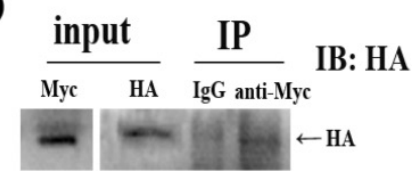

Figure 5. Downregulation of $p-p 38 \alpha$ protein level may be induced by a direct interaction between NLS-RAR $\alpha$ and $p 38 \alpha$ protein. (A) Immunofluorescence microscopy of NLS-RAR $\alpha$ staining with FITC(green) and $p 38 \alpha$ protein staining with TRITC(red) in NLS-RARa-overexpressed NB4 cells; (B, C) NLS-RARa was immunoprecipitated by anti-RAR $\alpha$, p38 $\alpha$ protein(Myc-tagged) was detected by Western blot; (D) p38 $\alpha$ was immunoprecipitated by anti-Myc, NLS-RAR $\alpha$ (HA-tagged) was detected by Western blot. IgG was used as control antibody. The overexpressions of $\mathrm{p} 38 \alpha$ protein and NLS-RARa(input) were determineded with Western blot analysis.

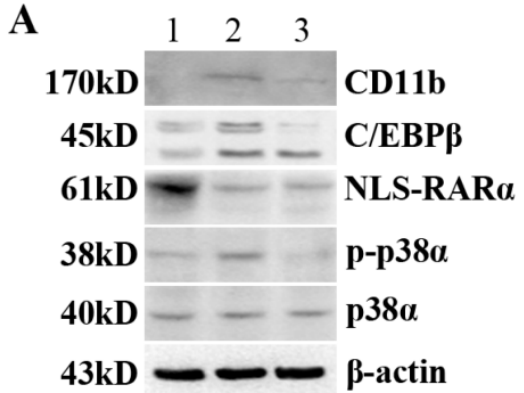

C

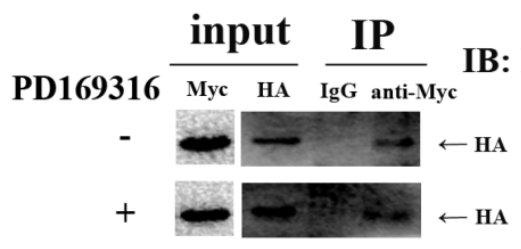

B

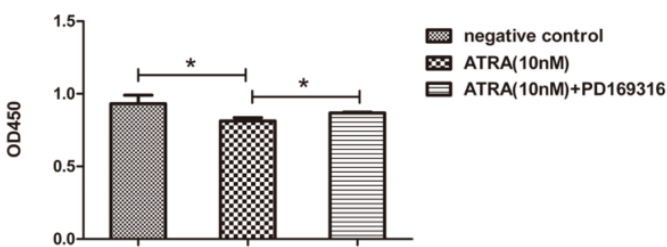

D

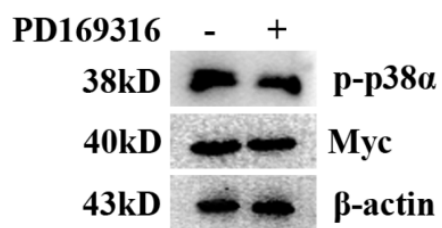

Figure 6. Recruitment $p 38 \alpha$-combinded NLS-RAR $\alpha$ by ATRA eventually caused activation of p38a protein. (A) Western blot analysis of the expressions of p38a, p-p38a, NLS-RAR $\alpha, C / E B P \beta$ and CD1 lb proteins in cultured NLS-RARa-overexpressed NB4 cells after dealing cells with various treatments for 3 days. (B) The proliferation of NLS-RARa-overexpressed NB4 cells in different groups was determined with CCK-8 Kit. (C) NLS-RARa(HA-tagged) was co-immunoprecipitated by anti-Myc in different groups. (D) The expressions of $\mathrm{p} 38 \alpha$ protein(Myc-tagged) and $\mathrm{P}-\mathrm{p} 38 \alpha$ protein in different groups. All data are presented as mean \pm SD. $*$ P $<0.05$. (1: negative group(DMSO-treated); 2: experimental group treated with $10 \mathrm{nM}$ ATRA; 3: experimental group treated with $10 \mathrm{nM}$ ATRA+10 $\mu$ M PD169316) 


\section{Discussion}

APL is a characteristic type of AML that originates from stem cells in the hematopoietic system of a clonal malignant disease. It is characterized by an unusually aggressive clinical course [27]. In our previous study, we have found that NLS-RARa and PML (NLS(-)) resulting from NE-induced PML-RARa cleavage could accelerate APL[7, 8, 28]. In this study, we further investigated the effects of NLS-RARa on NB4 cells and its potential mechanism.

ATRA is an accepted therapeutic drug for APL because it can promote cell differentiation while inhibit cell proliferation. Moreover, the effects of ATRA have been correlated with activation of p38a MAPK $[13,15,16]$. Here, we found that ATRA could promote differentiation while inhibit proliferation of NB4 cells via up-regulating the expression of p-p38a protein rather than the expression of p38a protein (Figure 2, 3). However, it didn't mean the more p-p38a protein expressed, the better for NB4 cells, since we observed a higher expression of CD11b protein and more effective inhibition on cell proliferation while lower expression of p-p38a protein when NB4 cells were treated with1 $\mu \mathrm{M}$ ATRA(Figure 2). Furthermore, dual luciferase reporter assay suggested that p-p38a protein has transcriptional activity (Figure 3). Interestingly, it has been reported that $\mathrm{p} 38 \mathrm{a}$ protein could interact with RARa directly then inhibit transcriptional activity of RARa in the presence of ATRA [13]. So we thought, p-p38a protein may likely compete with RARa for retinoid-responsive element.

In this study, we also revealed that NLS-RARa could inhibit differentiation while accelerate proliferation of NB4 cells via down regulating the expression of p-p38a protein in the presence of ATRA (Figure 4). Moreover, NLS-RARa could interact with p38a protein directly independent of ATRA (Figure 5). Those suggested that the underlyng mechanism of the effects of NLS-RARa on NB4 cells may be that ATRA recruited p38a-combinded NLS-RARa and then activated p38a protein while p38a-combinded NLS-RARa inhibited the effects of ATRA in the process (Figure 6). Thus, we supposed that patients with APL who are sensitive to ATRA may experience accelerated APL due to an NLS-RARa-induced downregulation of p-p38a protein, while NLS-RARa could promote APL progression by another pathway (e.g., Akt pathway) in patients with APL who are resistant to ATRA. However, it is unclear why there was a consistent variation between the expressions of $\mathrm{C} / \mathrm{EBP} \beta$ and $\mathrm{p}-\mathrm{p} 38 \mathrm{a}$ protein, even though both myeloid differentiation marker proteins, $\mathrm{C} / \mathrm{EBP} \beta$ and CD11b, increased after dealing NB4 cells with ATRA
(Figure 2). Moreover, pathogenesis of APL is still needed to be explored through experiments conducting in ATRA resistant APL cell lines, animal models of APL, and clinical samples.

\section{Abbreviations}

NLS-RARa: nuclear localization signal retinoic acid receptor alpha; NE: neutrophil elastase; PML-RAR $\alpha$ : promyelocytic leukemia-retinoic acid receptor alpha; APL: acute promyelocytic leukemia; MAPK: mitogen-activated protein kinase; C/EBP $\beta$ : CCAAT/enhancer binding protein beta; CD11b: integrin subunit alpha M; ATRA: All-trans Retinoic Acid; p-p38aMAPK: phosphorylated p38aMAPK; DMSO: dimethylsulfoxide; CCK-8: Cell Counting Kit-8; OD450: Optical density value at $450 \mathrm{~nm}$ wavelength; RARE: retinoic acid responsive element; LV-NC-NB4: NB4 cells were infected with lentivirusonly; LV-NLS-RARa-NB4: NB4 cells were infected with NLS-RAR-lentivirus; PBS: phosphate-buffered-saline; PMSF: phenylmethanesulfonyl fluo-ride.

\section{Acknowledgement}

The present study was supported by a grant from the National Natural Science Foundation of China (grant no. 81171658) and the Natural Science Foundation Project of CQ CSTC (grant no. 2011BA5037).We are appreciated professor Dmitrii Kamashev and his partners from France for favoring retinoid-responsive reporter construct of pTK-Luc.

\section{Conflicts of Interest}

The authors declare no conflict of interest.

\section{References}

1. Yoo SJ, Seo EJ, Lee JH, et al. A complex, four-way variant $t(15 ; 17)$ in acute promyelocytic leukemia. Cancer genetics and cytogenetics. 2006; 167: 168-71.

2. Kamimura T, Miyamoto T, Harada M, et al. Advances in therapies for acute promyelocytic leukemia. Cancer science. 2011; 102: 1929-37.

3. Nitto T and Sawaki K. Molecular Mechanisms of the Antileukemia Activities of Retinoid and Arsenic. Journal of Pharmacological Sciences. 2014; 126: 179-185

4. Kakizuka A, Miller WH, Jr., Umesono K, et al. Chromosomal translocation $t(15 ; 17)$ in human acute promyelocytic leukemia fuses RAR alpha with a novel putative transcription factor, PML. Cell. 1991; 66: 663-74.

5. Lane AA and Ley TJ. Neutrophil elastase cleaves PML-RARalpha and is important for the development of acute promyelocytic leukemia in mice. Cell. 2003; 115: 305-18.

6. Lane AA and Ley TJ. Neutrophil elastase is important for PML-retinoic acid receptor alpha activities in early myeloid cells. Molecular and cellular biology. 2005; 25: 23-33.

7. Hu XX, Zhong L, Zhang X, et al. NLS-RARalpha promotes proliferation and inhibits differentiation in HL-60 cells. International journal of medical sciences. 2014; 11: 247-54.

8. Song H, Li L, Zhong L, et al. NLS-RARalpha regulates the proliferation of leukemia cell NB4 by activating Akt pathway. Basic\&Clinical Medicine(China). 2016; 36: 41-46.

9. Kale VP. Differential activation of MAPK signaling pathways by TGF-beta1 forms the molecular mechanism behind its dose-dependent bidirectional effects on hematopoiesis. Stem cells and development. 2004; 13: 27-38.

10. Cabane C, Coldefy AS, Yeow K, et al. The p38 pathway regulates Akt both at the protein and transcriptional activation levels during myogenesis. Cellular signalling. 2004; 16: 1405-15. 
11. Alisi A, Spaziani A, Anticoli S, et al. PKR is a novel functional direct player that coordinates skeletal muscle differentiation via p38MAPK/AKT pathways. Cellular signalling. 2008; 20: 534-42.

12. Serra C, Palacios D, Mozzetta C, et al. Functional interdependence at the chromatin level between the MKK6/p38 and IGF1/PI3K/AKT pathways during muscle differentiation. Molecular cell. 2007; 28: 200-13.

13. Gianni M, Peviani M, Bruck N, et al. p38alphaMAPK interacts with and inhibits RARalpha: suppression of the kinase enhances the therapeutic activity of retinoids in acute myeloid leukemia cells. Leukemia. 2012; 26: 1850-61.

14. Watts JM and Tallman MS. Acute promyelocytic leukemia: what is the new standard of care? Blood reviews. 2014; 28: 205-12.

15. Qian X, He J, Zhao Y, et al. Inhibition of p38 MAPK Phosphorylation Is Critical for Bestatin to Enhance ATRA-Induced Cell Differentiation in Acute Promyelocytic Leukemia NB4 Cells. American journal of therapeutics. 2016; 23 : 680-89.

16. Wang R, Xia L, Gabrilove J, et al. Sorafenib Inhibition of Mcl-1 Accelerates ATRA-Induced Apoptosis in Differentiation-Responsive AML Cells. Clinical cancer research : an official journal of the American Association for Cancer Research. 2016; 22: 1211-21.

17. Duprez E, Wagner $\mathrm{K}$, Koch $\mathrm{H}$, et al. C/EBPbeta: a major PML-RARA-responsive gene in retinoic acid-induced differentiation of APL cells. The EMBO journal. 2003; 22: 5806-16.

18. Yamanaka R, Lekstrom-Himes J, Barlow C, et al. CCAAT/enhancer binding proteins are critical components of the transcriptional regulation of hematopoiesis (Review). International journal of molecular medicine. 1998; 1: 213-21.

19. Zeng $\mathrm{C}, \mathrm{Xu} \mathrm{Y}, \mathrm{Xu} \mathrm{L}$, et al. Inhibition of long non-coding RNA NEAT1 impairs myeloid differentiation in acute promyelocytic leukemia cells. BMC cancer. 2014; 14: 693.

20. Wang $Y$, Jin $W$, Jia $X$, et al. Transcriptional repression of CDKN2D by PML/RARalpha contributes to the altered proliferation and differentiation block of acute promyelocytic leukemia cells. Cell death \& disease. 2014; 5: e1431.

21. Chambon P. A decade of molecular biology of retinoic acid receptors. FASEB journal : official publication of the Federation of American Societies for Experimental Biology. 1996; 10: 940-54.

22. Minucci $S$ and Ozato $K$. Retinoid receptors in transcriptional regulation. Current opinion in genetics \& development. 1996; 6: 567-74.

23. Kamashev D, Vitoux D and De The H. PML-RARA-RXR oligomers mediate retinoid and rexinoid/cAMP cross-talk in acute promyelocytic leukemia cell differentiation. The Journal of experimental medicine. 2004; 199: 1163-74.

24. Rachez C and Freedman LP. Mediator complexes and transcription. Current opinion in cell biology. 2001; 13: 274-80.

25. Rastinejad F. Retinoid X receptor and its partners in the nuclear receptor family. Current opinion in structural biology. 2001; 11: 33-8.

26. Kambhampati S, Verma A, Li Y, et al. Signalling pathways activated by all-trans-retinoic acid in acute promyelocytic leukemia cells. Leukemia \& lymphoma. 2004; 45: 2175-85.

27. Lo-Coco F and Cicconi L. History of acute promyelocytic leukemia: a tale of endless revolution. Mediterranean journal of hematology and infectious diseases. 2011; 3: e2011067.

28. Yang XQ, Wang H, Jiang KL, et al. The effects of PML ( NLS -) on leukemia cell NB4 mediated by adenovirus-over-expression. Basic \& Clinical Medicine(China). 2014; 34: 1327-32. 